\title{
UNDER THE LENS
}

\section{A spike with which to beat COVID-19?}

\author{
Nawsad Alam and Matthew K. Higgins
}

This month's Under the Lens discusses how structural studies of the SARS-CoV-2 spike glycoprotein might guide a path towards a vaccine.

As SARS-CoV-2 (the cause of the COVID-19 pandemic) has swept across the globe, we have been reminded of what it is like to live with rampant infectious disease, without effective drugs or vaccines. Researchers have piled in to the quest to provide rational designs. The structural biology community are no exception, with structures of SARS-CoV-2 proteins emerging at unprecedented speed. One of their main focuses has been the viral spike glycoprotein that binds to the receptor angiotensin-converting enzyme 2 (ACE2), allowing virus entry. Here, we discuss some of these recent papers on structural characterization of the spike and highlight how they might guide structure-based approaches to develop an effective spike-based vaccine.

Coronavirus spikes are trimeric molecules that present their receptor-binding domains (RBDs) at their distal tips. These RBDs recognize receptors, allowing the virus to enter cells. During entry, the spike undergoes a large conformational rearrangement from its pre-fusion to post-fusion state, driving viral fusion with the cell membrane. Coronavirus spikes have been structurally characterized before, in particular for SARS-CoV and MERS-CoV, two related viruses that have caused epidemics of respiratory disease. A crucial question was whether SARS-CoV-2 resembled either of these related viruses. If it did, this would be exciting news, as years of study of these viruses could be applied to the quest for a SARS-CoV-2 vaccine.

Using cryo-electron microscopy, a number of studies, including Walls et al. ${ }^{1}$, determined structures of the spike. These resembled most closely the structure of the SARS-CoV spike. As seen for other coronaviruses, two conformations were visualized a closed state, with the RBDs docked against the trimeric core, and an open conformation with a single RBD raised into its 'up' position'. These studies also showed, just like SARS-CoV, that SARS-CoV-2
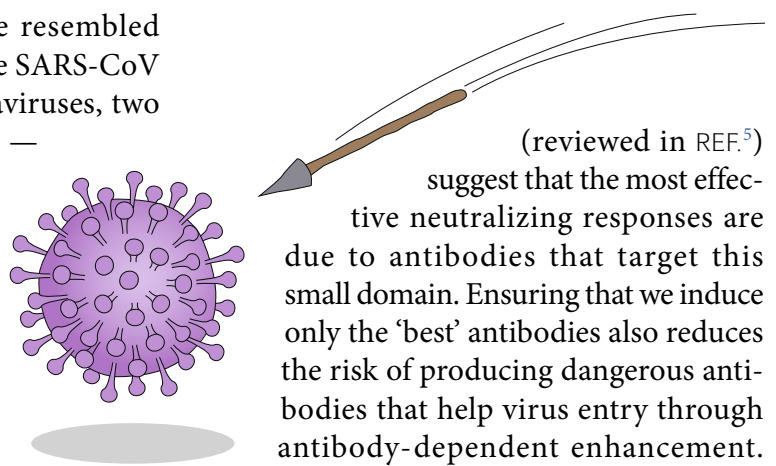

tive neutralizing responses are due to antibodies that target this small domain. Ensuring that we induce only the 'best' antibodies also reduces the risk of producing dangerous antibodies that help virus entry through antibody-dependent enhancement. Whether the future holds stabilized entry $^{1}$. A crystal structure soon followed, showing how the RBD binds to ACE2 (REF. ${ }^{2}$ ). Shortly after this, the first structure of a neutralizing antibody bound to the RBD of the spike glycoprotein showed that SARS-CoV-reactive antibodies could neutralize the new virus ${ }^{3}$, and polyclonal antibody sera against SARS-CoV also proved effective ${ }^{1}$. In many ways, these structural studies showed that the new virus resembles a virus that causes SARS; a true SARS-CoV-2.

So, what does this mean for design of a vaccine? Two spike-based vaccine-design strategies, developed for SARS-CoV, should be applicable. The first approach relies on stabilization of the spike. To generate the most effective neutralizing antibody responses, vaccinated individuals should generate antibodies to the pre-fusion spike, thus preventing ACE2 binding and cell entry. For this reason, structure-guided mutations have been designed that fix the SARS-CoV spike into this pre-fusion state ${ }^{4}$. Stabilized SARS-CoV-2 spikes, designed using this method, are therefore promising for a vaccine. But do we need the whole spike? A second approach is to design a vaccine that just contains the RBD. A number of studies spikes or RBDs, the new spike structures, which revealed that our knowledge of SARS$\mathrm{CoV}$ is also applicable to SARS-CoV-2, are good news. Now we must all hope that the quest to design rational vaccines based on this knowledge is as quickly realized as those structural studies, as the research community work together to fight this global pandemic.

\section{Nawsad Alam and Matthew K. Higgins ${ }^{\square}$ Department of Biochemistry, University of Oxford Oxford, UK. \\ 凶e-mail: underthelens@bioch.ox.ac.uk https://doi.org/10.1038/s41579-020-0383-2}

1. Walls, A. et al. Structure, function, and antigenicity of the SARS-CoV-2 spike glycoprotein. Cell 180 281-292 (2020).

2. Shang, J. et al. Structural basis of receptor recognition by SARS-CoV-2. Nature 581, 221-224 (2020).

3. Yuan, M. et al. A highly conserved cryptic epitope in the receptor-binding domains of SARS-CoV-2 and SARS-CoV. Science 368, 630-633 (2020).

4. Kirchdoefer, R. N. et al. Stabilized coronavirus spikes are resistant to conformational changes induced by receptor recognition or proteolysis. Sci. Rep. $\mathbf{8}$, 15701 (2018).

5. Jiang, S. et al. Receptor-binding domains of spike proteins of emerging or re-emerging viruses as targets for development of antiviral vaccines. Emerg. Microbes Infect. 1, e3 (2012).

Competing interests

The authors declare no competing interests. 